\title{
Periprosthetic Femoral Fractures in Total Hip Arthroplasty (THA): Comparison Between Osteosynthesis and Revision
}

Gianluca Scalici ( $\sim$ gianlucascalici1991@gmail.com ) AOU Careggi: Azienda Ospedaliero Universitaria Careggi https://orcid.org/0000-0002-5480-0439

Debora Boncinelli AOU Careggi: Azienda Ospedaliero Universitaria Careggi

Luigi Zanna

AOU Careggi: Azienda Ospedaliero Universitaria Careggi

Roberto Buzzi

AOU Careggi: Azienda Ospedaliero Universitaria Careggi

Laura Antonucci

AOU Careggi: Azienda Ospedaliero Universitaria Careggi

\section{Fabrizio Di Maida}

AOU Careggi: Azienda Ospedaliero Universitaria Careggi

Pietro De Biase

AOU Careggi: Azienda Ospedaliero Universitaria Careggi

\section{Research article}

Keywords: Periprosthetic fractures, Hip arthroplasty, Revision Arthroplasty, CIRS score

Posted Date: May 13th, 2021

DOl: https://doi.org/10.21203/rs.3.rs-482086/v1

License: (9) (i) This work is licensed under a Creative Commons Attribution 4.0 International License. Read Full License 


\section{Abstract}

Background Periprosthetic fractures are challenging complication of hip arthroplasty and a rare injury, but their incidence is increasing. The surgical treatment is demanding to achieve early mobilization and avoid the complications of prolonged recumbency. The aim of this study is to evaluate the clinical outcomes of surgical treatment in periprosthetic femoral fractures of hip arthroplasty, making a comparison between implant revision and reduction and internal fixation of the fracture.

Methods Authors retrospectively reviewed a series of 117 patients with total hip arthroplasty treated for periprosthetic femur fractures in the period between January 2013 and March 2018 at a single tertiary referral center. We collected the data of 70 patients who satisfied inclusion criteria. The fractures were classified according to the Unified Classification System (UCS) and randomized in two groups: reduction and internal fixation (G1) or revision arthroplasty (G2). Clinical outcomes were assessed with Oxford Hip Score post and pre-surgery, Barthel Score in relation to CIRS score (Cumulative illness rating scale), the type of fracture and post-operative complications with a minimum follow up of one year.

Results Nominal univariable statistical analysis revealed significantly results concerning the difference between the post and pre-operative Oxford Hip Score ( $D$ Oxford) with type of treatment $(p=0,008)$ and with the CIRS score $(p=0,048)$. Moreover, we observed a strong significant relationship between type of treatment and type of fracture $(P=0,0001)$. Multivariable analyses revealed that CIRS score was independently associated with Oxford Score improvement after surgery $(P=0,024)$.

Conclusions Authors reported how the surgical revision has a better functional outcome in B2 type of fracture than $\mathrm{B} 1$ or $\mathrm{C}$, but the chosen treatment should be related to surgeon's experience and patient's comorbidities and a multidisciplinary team.

\section{Background}

Periprosthetic femoral fractures (PPFx) are challenging complications of hip arthroplasty for orthopedic surgeons. The increasing incidence is related to larger numbers of prosthetic replacement per year and higher life expectancy (1). PPFx are going to involve a significant amount of operating time, bed and cost days and are associated with both high mortality and morbidity. (2)(3) (Fig. 1)

According to current available literature and the Annual Report 2019 of the National Joint Replacement Registry (Australian Orthopedic Association), there has been a $124.9 \%$ increase in primary total conventional hip replacement procedures performed since 2003. Among these high numbers most frequent complications after primary total hip replacement are loosening (24.6\%), dislocation $(20.8 \%)$ and fracture as $20.7 \%$. Reports from single centers show a lower incidence. (4)(5)

Surgical treatment is turned to achieve early mobilization and avoid complications of prolonged bed rest. Achieving a stable fixation is demanding for to the interference of femoral stem with synthesis devices, osteoporotic bone quality and altered anatomy. PPFx result in severe morbidity for patients. Undergoing 
revision surgery for periprosthetic fractures results in a worse survival outcome than the general population at one year and that bias persists for several years after surgery. (6)(7)(8). Non-operative management is not practicable as for complications from long-lasting period of immobilization and potential fracture non-union when there is a cemented stem (9). Main features implicated are location of the fracture, type of prosthetic fixation, cemented or cementless and adequacy of bone stock and supporting the implant.

In the present study we assessed the surgical and clinical postoperative outcomes of patients, treated for periprosthetic fractures after total hip arthroplasty comparing open reduction and internal fixation (ORIF) or revision arthroplasty (RA) of the femoral stem.

\section{Methods}

The Authors retrospectively reviewed a series of 117 patients with total hip arthroplasty treated for PPFx between January 2013 and March 2018, who presented to our hospital (single tertiary referral center). The patients who had associated fractures, the fracture pattern and the injury's severity score were considered specifically.

The exclusion criteria were conservative management, minimum available follow-up lower than 12 months, undetectable and incomplete clinical or radiological records for cases referred from other hospital, intraoperative fractures, fractures related to tumoral lesions and concomitant infection. This left 68 cases, but 70 fractures because two patients presented with bilateral fractures.

Clinical and demographic data (gender, age, body mass index, side of fracture) were retrospectively gathered from clinical records. The highlighted data, model of femoral component and/or plate used, type of surgical technique used, number of cerclage wires and blood transfusion are collected in clinical records (digitally stored). Moreover, Authors used the CIRS score (Cumulative illness rating scale) to assess the comorbidity level of the patients before surgery. This scoring system measures the chronic medical illness taking into consideration the severity of chronic disease in 14 items. Each item has a 0-4 score, where 0 represent "no problem affecting that system" and 4 "extremely severe problem and/or immediate treatment required and /or organ failure and/or severe functional impairment". The cumulative final score can vary from 0 to 56 . (10)

The Unified Classification System (UCS) for PPFx, based on the Vancouver Classification relating to the femur fractures and described by Duncan and Masri in 1995 (11) was employed in the study (14) and it was confirmed on the intra-operative evaluation performed during surgical procedures.

The patients were randomized to two groups (G1 and G2) depending on the surgical treatment, ORIF vs RA. Clinical features and overall post-operative complications (infection, hardware failure, dislocations, non-union, deep venous thrombosis, heart failure and pneumonia) were analyzed in relation to fracture classification and type of surgical management (ORIF or RA) with a minimum follow up of one year. Pre- 
and post- surgical clinical outcomes and disability of patients were evaluated respectively with Oxford Hip score and Barthel score.

Different surgical procedures have been performed considering the type of fracture, the quality of the bone stock, the general conditions of the patient before the fracture with adequate functional and cognitive assessment and the associated comorbidities. (Fig. 2)

When the prosthetic implants were considered stable (Duncan Classification type B1 or C) ORIF was performed; the bone stock should be adequate for synthesis and clinical conditions of the patients should allow a longer recovery and rehabilitation times. Prosthetic revision was used in case of loosening of prosthesis stability (type B2), inadequate bone stock (type B3) or when general conditions of the patient required a rapid mobilization and ambulation. Cerclage wires were added to RA procedures to provide adequate support for revision stem and avoid new fractures during revision procedures. We routinely used the postero-lateral access to the hip with lateral extension to proximal femur, which in our experience allows a good exposure of the fracture pattern and facilitates a direct reduction. In every case surgery was performed by a senior surgeon with adequate experience in trauma or revision surgery.

Descriptive statistics were obtained reporting medians and interquartile ranges (IQR) for continuous variables and frequencies and proportions for categorical variables, as appropriate. Continuous variables were compared by the Student Independent $T$ test or the Mann-Whitney $U$ test based on their normal or not-normal distribution, respectively. Normality of variables' distribution was tested by the KolmogorovSmirnov test. Categorical variables were tested with the Chi-square and Kruskal-Wallis tests. Differences between pre- and post-treatment variables were assessed using paired $T$ test. Univariable and multivariable ANOVA (MANOVA) with post hoc analysis was adapted to compare clinical and surgical variables between different types of treatment. Multivariable analysis was performed to explore predictors of $\Delta$ Oxford, after adjusting for age, preoperative Barthel and CIRS score. Statistical significance was set as $p<0.05$. All tests were two-sided. Analyses were carried out using SPSS v. 24 (IBM SPSS Statistics for Mac, Armonk, NY, IBM Corp)

\section{Results}

The study cohort included 70 considered eligible from 117 patients evaluated. The mean age of patients at the time of fracture was 88 years (IQR, 83-96 years) and the median age 90 years (SD $\pm 10,21) .55$ patients were female (78.6\%) and 15 males (21.4\%). In 41 cases the affected side was right $(58.6 \%)$ and in 29 left (41.4\%). The median of Oxford Score Pre-Surgery was 36 (IQR 22-43, SD +-12.21) and the median of Barthel Pre-Surgery score was 87.5 (IQR, 65-100; SD +-22.35). Moreover, the comorbidity median score of the series, evaluated with CIRS score, was eight (IQR, 5-11; SD +- 4.35). Preoperative data are reported in Table 1.

According to UCS classification, the most frequent type of fracture was B2 (32 cases, $45.7 \%$ ) followed by C (22 cases, 31.4\%), B1 (13 cases, 18.6\%) and B3 (3 cases, 4.3\%). Patients were divided in two groups: G1 treated with ORIF (Fig. 3) and G2 treated with RA (Fig. 4). G1 patients (45 cases, 64.3\%) were treated with 
ORIF with Non-Contact Bridging Periprosthetic Femur Plate System (NCBâ PFP, Zimmer) in 40 patients and Less Invasive Stabilization System plates (LISS, Synthes) in five patients; implant revision Wagner SL Revisionâ Hip Stem (Zimmer) plus ORIF was performed in two cases. In G2 patients (25 cases, 35.7\%) RA with revision Wagner SLâ prosthetic stem was performed. Cerclage wires were added in 51 cases $(72.9 \%)$ to achieve adequate reduction prior to ORIF or RA. Operative procedures are detailed in Table 2.

During hospitalization 40 patients $(62.5 \%)$ required blood transfusions due to post-surgery anemia. Postoperative venous ultrasound (VUS) based on B-mode, combined with color-Doppler US and power imaging techniques on the fifth post-operative day recorded in seven cases $(10.9 \%)$ the presence of a deep vein thrombosis of the lower limb. This was the most frequent post-operative complication, along with seven cases of congestive heart failure (10.9\%) and five cases of pneumonia (7.8\%) treated with intravenous antibiotics. Moreover, in three patients (4.7\%) occurred implant aseptic loosening, in two cases (3.1\%) an implant prosthetic infection and in one patient (1.6\%) a THA posterior dislocation during the follow up. These six patients required an additional treatment and implant revision. Post-operative data are reported in Table 2.

The median Oxford Post-Surgery Score was 32 (IQR, 19-40; SD +- 12.07) and the median of Barthel Post Surgery Score was 77.5 (IQR, 46.25-90; SD +-26.6). (Table 2).

Results of the univariable analyses between pre-operative, operative and post-operative data in relation to the type of treatment, reduction and synthesis or prosthetic revision are reported in the Table 3. Preoperative, operative and post-operative features, like gender $(p=0.474)$, side $(p=0.162)$, blood transfusion $(p=0.538)$ and using of cerclages $(p=0.717)$ were not significantly associated with the type of treatment. As well, the incidence of post-operative complications, deep vein thrombosis, vascular damage, nonunions or consolidation delay and infections, does not statistically significantly correlate with the surgical treatment performed ( $p=0.936)$, ORIF vs. RA (Table 3). Nominal univariable analyses resulted in significantly differences between the post-operative and pre-operative Oxford Hip Score with the type of treatment $(p=0.008)$ and with the CIRS score $(p=0,048)$; other univariable analyses did not give significantly results (Table 3). Moreover, the relationship between the type of treatment and the type of fractures was highly highlighted extremely strong $(p=0.0001)$.

MANOVA with post hoc analysis showed a significant difference according to $D$ Oxford between patients undergoing reduction and synthesis or prosthesis revision $(p=0.042)$.

At multivariable analysis, after adjusting for age, preoperative Barthel and type of fracture assessed by Duncan classification, the CIRS score was independently associated with Oxford Score improvement after surgery $(p=0.024)$, while type of treatment was not.

\section{Discussion}

The treatment of periprosthetic femoral fractures (PPFx) is really challenging for orthopaedic surgeon. These fractures require often senior surgeons with competence both in ORIF and RA. Also the patients 
itself are challenging for usually present severe comorbidities (12)(13). The treatment of PPFx has been associated with high risk of failure, poor outcomes and worst survival outcome compared to general population at one year and this item persists for five-eight years after surgery (8)(14).

The treatment goals are restoring stem stability and limb alignment, recovery of pre-fracture functional mobility and early mobilization. In B1 and C fracture types advocated standard treatment is ORIF, but in older patients it could be demanding due to weakened bone quality and potential bone loss (6)(15). Recommended treatment of B2 and B3 fractures is femoral stem revision, eventually reinforced with plating or isolated cerclage wires, using long stems with diaphyseal fixation to achieve a more stable construct. (1)(16)

In this study, a strong statistic correlation was found between the type of fracture, according to UCS Periprosthetic Fractures classification, and the surgical treatment used, conforming the main indications of treatment.

Authors evaluated the clinical outcome based on difference of Oxford Score post and pre-surgery, after RA compared to ORIF of the periprosthetic fractures. The univariate analysis showed a better clinical post-operative outcome of patients treated with prosthetic revision (B2) instead of those treated with ORIF (B1 or C); these data disagree with current literature that did not demonstrated a different functional outcome depending on treatment modality (17). To better address this topic, we performed a statistical analysis between functional outcome of ORIF in B1 or $\mathrm{C}$ fracture cases, with relatively similar groups, and found no significant differences $(p=0,143)$. Therefore, RA better outcomes are not related to abnormal indications to ORIF but relates to better functional postoperative status of patients treated with revision stems, underlying that revision, when indicated, can predict a better outcome in these fragile patients. These results are not completely aligned to literature data but are not related to ORIF technique or stem stability. (18)

Although the functional outcome is closely related with the type of surgical treatment, the multivariable analysis of this study reveals also that is influenced by the patient's age, pre-operative Barthel Score and the CIRS score.

The level of clinical outcome, based on the Delta Oxford Score (post- and pre-operative), has a statistical relation with CIRS score, even if this correlation is not linear. The CIRS score is a questionnaire based on the various comorbidities of the patient that have a different influence on the post-operative rehabilitation and functional outcome, such as the respiratory disease compared to gastrointestinal disorders, but they all result in the same score. Consequently, Authors recorded different Delta Oxford Score in patients with same CIRS score. The association between CIRS score and Delta Oxford score is also related to the age, pre-operative Barthel score and type of fractures but does not correlate with the surgical treatment.

In literature data survival after PPFx is worse than any other cause of revision THA, like infection, aseptic loosening and dislocation. Cnudde et al (8) found that survival in the repeated surgery after THA is influenced by the reason for re-operation, and PPFx have a five-year survival rate of $54 \%$, worse than the 
rates of RA for different complications. (19) Young et al. (20) analysed the functional outcome of revision for periprosthetic fractures and found that patients had poorer outcome and higher death rates compared to those undergoing revision THA for aseptic loosening. On the same side Young underline also the lower mortality rate in patient who had been operated by experienced surgeons and at larger centers.

According to literature, the patient mortality rates at one year remain high (13-17\%) despite improvements in surgical and fixation techniques (15)(21). However, Bhattacharyya et al. (22) reported that one year mortality rate in patients treated for type $B$ fractures with internal fixation was $33 \%$ while those treated with femoral stem revision experienced a $12 \%$ mortality rate. Tucker et al reported that Vancouver classification is an accurate system to choose the surgical treatment but is not correlated with the 12 months mortality rates, nor was the length of surgical procedure (23).

Stoffel et al. (18) compared the functional outcomes of patients undergoing ORIF or RA in Vancouver type B2 and B3 PPFx. Their study highlighted that ORIF could be a viable treatment option considering the type of prosthetic stem, anatomical reduction of fracture and intact cement mantle.

\section{Conclusions}

This retrospective study collects a large series of patients treated in a single high-volume center both for trauma and arthroplasty surgeries. Every case was treated by a senior surgeon with large experience both in RA and ORIF. Authors did not find differences between internal fixation and revision arthroplasty in terms of blood loss and reported how the RA revision has a better functional outcome in B2 type of fractures than the B1 or C, but the chosen treatment needs to be related to surgeon's experience and the patient's compliance. In accordance with our study, it underlines the importance of suitable surgical treatment in relation to the type of fracture and it opens new considerations regarding new surgical options compared to the usual treatment algorithm.

The limits of this study are the retrospective form, but it demonstrated some noteworthy data deserving a prospective study. Our results are also influenced by being collected from a single department, both in negative and positive means. Decision making was always reviewed by collegial meeting and choice of treatment was constant throughout the period of study.

\section{List Of Abbreviations}

Periprosthetic femoral fractures in Total Hip Arthroplasty (THA)

Unified Classification System (UCS)

Cumulative illness rating scale (CIRS)

Periprosthetic femoral fractures (PPFx)

Open reduction and internal fixation (ORIF) 
Revision arthroplasty (RA)

Interquartile ranges (IQR)

\section{Declarations}

Ethical approval and consent to participate: This study was approved by our institutional review board; Informed consent was obtained from all patients for being included in the study.

Consent for publication: Informed consent was obtained from all patients for being included in the study.

Availability of data and materials: the datasets generated and/or analyzed during the current study are not publicly available due but are available from the corresponding author on reasonable request, because there are presents private data of the patients.

Competing interests: The authors declare that they have no conflict of interest. Not applicable.

Funding: Nothing material or monetary was received by any of the authors in the execution of this study and the subsequent production of this manuscript. Not applicable.

Author's contributions: GS, DB, LZ and PdB as designers of the study.

GS, DB, LA, FdM for acquisition, analysis and intepretation of data. GS, DB, RB e PdB as revisors of the study.

All Authors have read and approved the manuscript.

Acknowledgements: Not applicable.

\section{References}

1. Canbora K, Kose O, Polat A, Aykanat F, Gorgec M. Management of Vancouver type B2 and B3 femoral periprosthetic fractures using an uncemented extensively porous-coated long femoral stem prosthesis. Eur J Orthop Surg Traumatol. 2013;23(5):545-52.

2. Abdel MP, Watts CD, Houdek MT, Lewallen DG, Berry DJ. Epidemiology of periprosthetic fracture of the femur in 32644 primary total hip arthroplasties: A 40-year experience. Bone $\mathrm{Jt} \mathrm{J}$. 2016;98B(4):461-7.

3. Chatziagorou G, Lindahl H, Garellick G, Kärrholm J. Incidence and demographics of 1751 surgically treated periprosthetic femoral fractures around a primary hip prosthesis. HIP Int. 2019;29(3):282-8.

4. Yasen AT, Haddad FS. Periprosthetic fractures: Bespoke solutions. Bone Jt J. 2014;96B(11):48-55.

5. Misur PN, Duncan CP, Masri BA. The Treatment of Periprosthetic Femoral Fractures After Total Hip Arthroplasty. JBJS Rev. 2014;2(8):1. 
6. Lee J-M, Hwang D-W, Choi H-J, Seol Y-D, Lee D-S. Treatment of Periprosthetic Femoral Fractures after Hip Arthroplasty. J Korean Orthop Assoc. 2010;45(2):114.

7. Cook RE, Jenkins PJ, Walmsley PJ, Patton JT, Robinson CM. Risk factors for periprosthetic fractures of the hip: A survivorship analysis. Clin Orthop Relat Res. 2008;466(7):1652-6.

8. Cnudde P, Bülow E, Nemes S, Tyson Y, Mohaddes M, Rolfson O. Association between patient survival following reoperation after total hip replacement and the reason for reoperation: an analysis of 9,926 patients in the Swedish Hip Arthroplasty Register. Acta Orthop. 2019;90(3):226-30.

9. Tsiridis E, Gie GA. Percutaneous plate fixation for periprosthetic femoral fractures - A preliminary report [1]. Injury. 2002;33(9):845.

10. Salvi F, Miller MD, Grilli A, Giorgi R, Towers AL, Morichi V, et al. A manual of guidelines to score the modified Cumulative IIIness Rating Scale and its validation in acute hospitalized elderly patients. J Am Geriatr Soc. 2008;56(10):1926-31.

11. Brady OH, Garbuz DS, Masri BA, Duncan CP. The reliability and validity of the Vancouver classification of femoral fractures after hip replacement. J Arthroplasty. 2000;15(1):59-62.

12. Registry AOANJR. Hip, Knee \& Shoulder Arthroplasty. Annual report 2019.

13. Lindahl H, Malchau H, Herberts P, Garellick G. Periprosthetic femoral fractures: Classification and demographics of 1049 periprosthetic femoral fractures from the Swedish National Hip Arthroplasty Register. J Arthroplasty. 2005;20(7):857-65.

14. Yao JJ, Kremers HM, Abde MP, Larson DR, Ransom JE, Berry DJ, et al. Long-term Mortality after Revision THA. Clin Orthop Relat Res. 2018;476(2):420-6.

15. Moreta J, Aguirre U, De Ugarte OS, Jáuregui I, Mozos JLMDL. Functional and radiological outcome of periprosthetic femoral fractures after hip arthroplasty. Injury. 2015;46(2):292-8.

16. Kim Y, Tanaka C, Tada H, Kanoe H, Shirai T. Treatment of periprosthetic femoral fractures after femoral revision using a long stem. BMC Musculoskelet Disord [Internet]. 2015;16(1):1-7. Available from: ???

17. Märdian S, Schaser KD, Gruner J, Scheel F, Perka C, Schwabe P. Adequate surgical treatment of periprosthetic femoral fractures following hip arthroplasty does not correlate with functional outcome and quality of life. Int Orthop. 2015;39(9):1701-8.

18. Stoffel K, Blauth M, Joeris A, Blumenthal A, Rometsch E. Fracture fixation versus revision arthroplasty in Vancouver type B2 and B3 periprosthetic femoral fractures: a systematic review. Arch Orthop Trauma Surg [Internet]. 2020;140(10):1381-94. Available from: https://doi.org/10.1007/s00402-020-03332-7

19. Fenelon C, Murphy EP, Kearns SR, Curtin W, Murphy CG. A growing challenge: The rise of femoral periprosthetic fractures - An 11-year observational study. Surgeon [Internet]. 2020;18(1):19-23. Available from: https://doi.org/10.1016/j.surge.2019.05.001

20. Young SW, Walker CG, Pitto RP. Functional outcome of femoral peri prosthetic fracture and revision hip arthroplasty: A matched-pair study from the New Zealand Registry. Acta Orthop. 2008;79(4):4838. 
21. Drew JM, Griffin WL, Odum SM, Van Doren B, Weston BT, Stryker LS. Survivorship After Periprosthetic Femur Fracture: Factors Affecting Outcome. J Arthroplasty [Internet]. 2016;31(6):1283-8. Available from: http://dx.doi.org/10.1016/j.arth.2015.11.038

22. Bhattacharyya $T$, Chang $D$, Meigs JB, Estok $D M$, Malchau $H$. Mortality after periprosthetic fracture of the femur. J Bone Jt Surg - Ser A. 2007;89 A(12):2658-62.

23. Finlayson G, Tucker A, Black ND, McDonald S, Molloy M, Wilson D. Outcomes and predictors of mortality following periprosthethic proximal femoral fractures. Injury [Internet]. 2019;50(2):438-43. Available from: https://doi.org/10.1016/j.injury.2018.10.032

\section{Tables}

Table 1. Pre-operative data of patients.

\begin{tabular}{|lll|}
\hline Preoperative Features & & \\
\hline Gender, n. (\%) & Male & $15(21,4 \%)$ \\
\cline { 2 - 3 } & Female & $55(78,6 \%)$ \\
\hline Age, median (IQR) & & $90(83-96)$ \\
\hline Side, n. (\%) & Right & $29(41,4 \%)$ \\
\hline Oxford Score Pre-Surgery, median (IQR) & $36(22-43)$ \\
\hline Barthel Score Pre- Surgery, median (IQR) & $87,5(65-100)$ \\
\hline CIRS Scale, median (IQR) & & $8(5-11)$ \\
\hline Duncan Classification UCS, n. (\%) & B1 (\%) & $13(18,6 \%)$ \\
\cline { 2 - 3 } & B2 (\%) & $32(45,7 \%)$ \\
\hline & B3 (\%) & $3(4,3 \%)$ \\
\hline & C (\%) & $22(31,4 \%)$ \\
\hline
\end{tabular}

Table 2. Operative and Post-operative data of patients. 


\begin{tabular}{|lll|}
\hline $\begin{array}{l}\text { Peri- operative } \\
\text { features }\end{array}$ & \\
\hline Type of Surgery, n. (\%) & Reduction and Synthesis (\%) & $45(64,3 \%)$ \\
\cline { 2 - 3 } & Prosthetic revision (\%) & $25(35,7 \%)$ \\
\hline Cerclages, n. (\%) & Yes (\%) & $51(72,9 \%)$ \\
\cline { 2 - 3 } & No (\%) & $19(27,1 \%)$ \\
\hline
\end{tabular}

\begin{tabular}{|lll|}
\hline $\begin{array}{l}\text { Post- operative } \\
\text { features }\end{array}$ & & \\
\hline Post-operative complications, n. (\%) & Absent (\%) & $39(60,9 \%)$ \\
\cline { 2 - 3 } & \multicolumn{1}{l}{ Deep Venous Thrombosis (\%) } & $7(10,9 \%)$ \\
\cline { 2 - 3 } & \multicolumn{1}{|c|}{ Aseptic loosening (\%) } & $3(4,7 \%)$ \\
\cline { 2 - 3 } & Dislocations (\%) & $1(1,6 \%)$ \\
\cline { 2 - 3 } & Pneumonia (\%) & $5(7,8 \%)$ \\
\cline { 2 - 3 } & Vascular injury (\%) & $7(10,9 \%)$ \\
\cline { 2 - 3 } & Infection (\%) & $2(3,1 \%)$ \\
\hline Blood Transfusion, n. (\%) & Yes (\%) & $40(62,5 \%)$ \\
\cline { 2 - 3 } & No (\%) & $24(37,5 \%)$ \\
\hline Oxford Score Post-Surgery, median (IQR) & $32(19,75-40,25)$ \\
\hline Barthel Score Post-Surgery, median (IQR) & $77,50(46,25-90)$ \\
\hline
\end{tabular}

Table 3. Univariable statistical analyses on patients' data. 


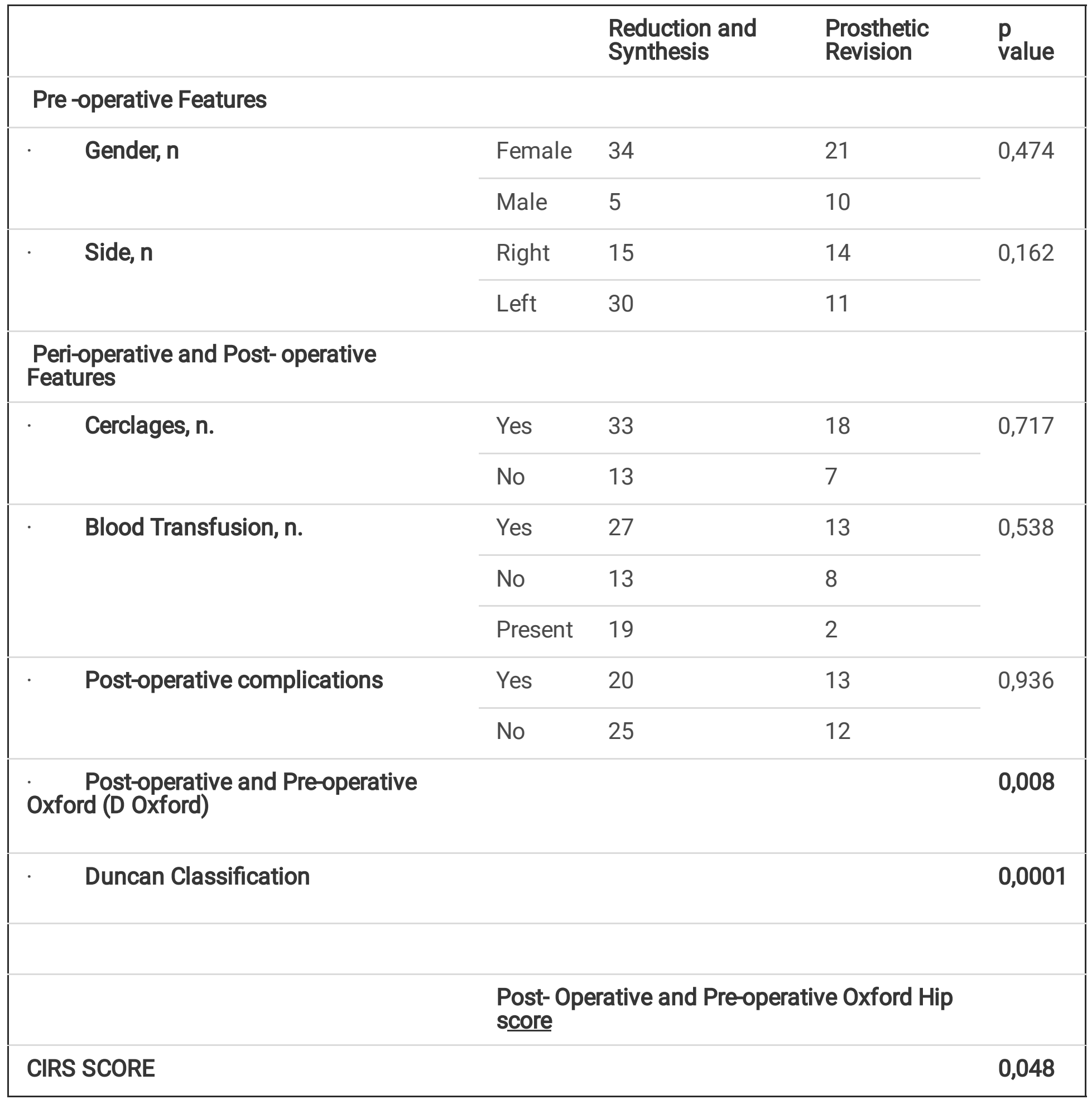

\section{Figures}




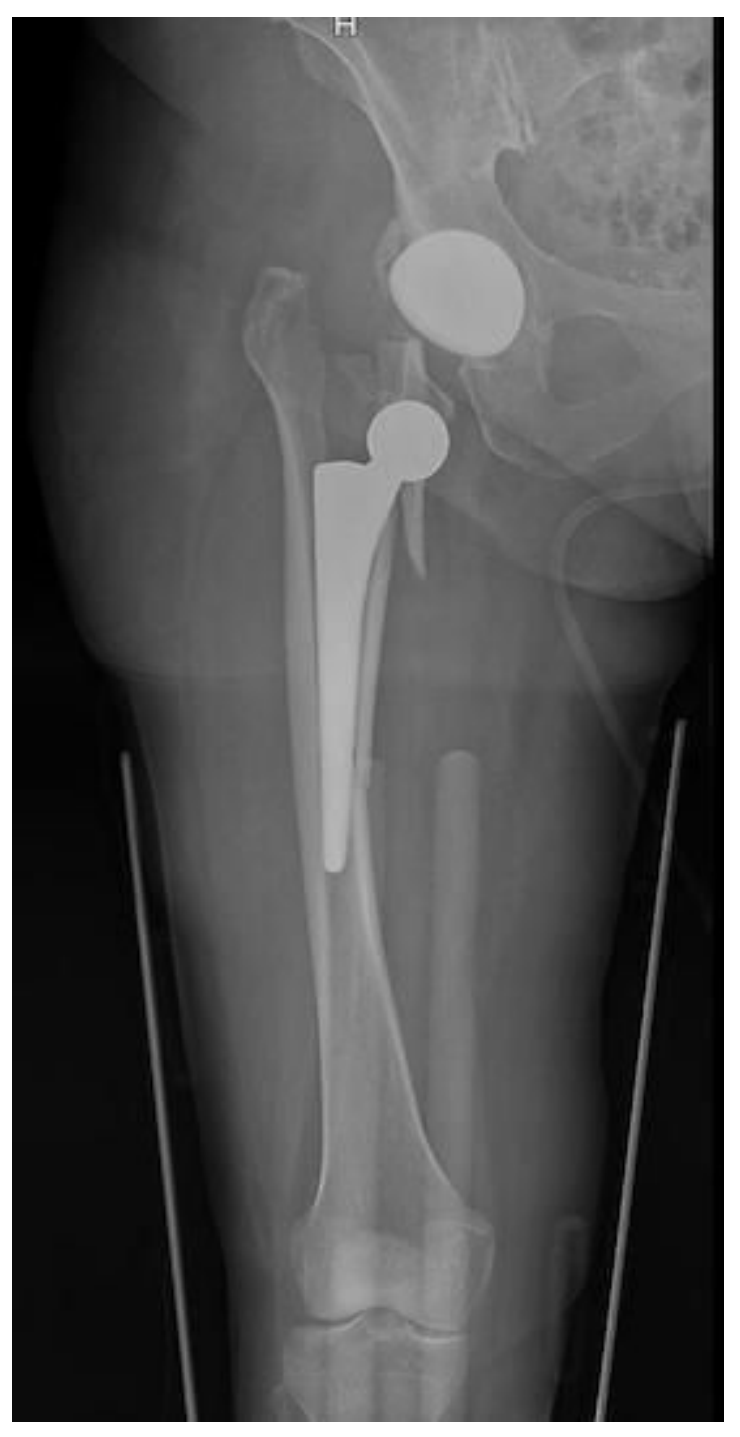

Figure 1

Periprosthetic femoral fracture with stem mobilization. 


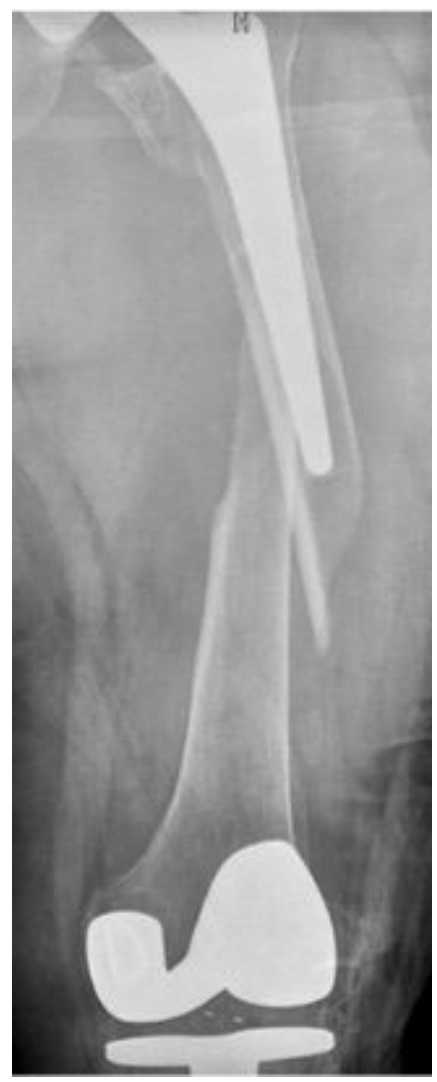

Figure 2

Distal stem periprosthetic femoral fracture.

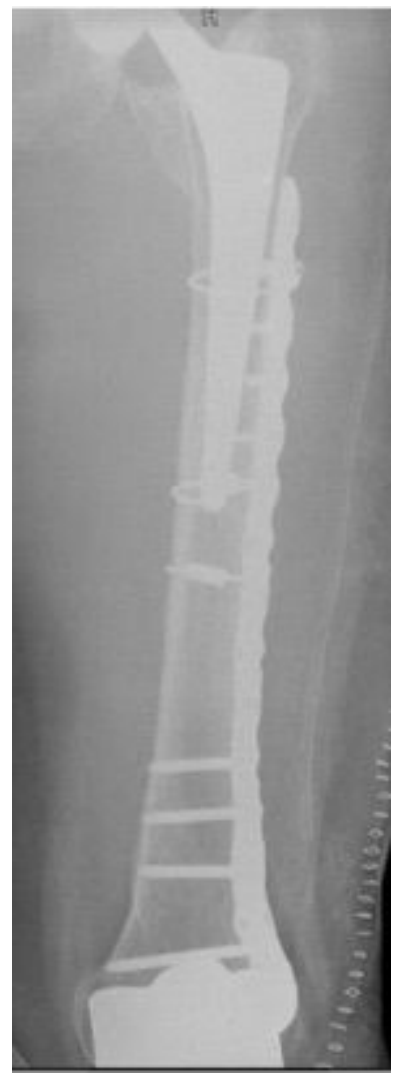

Figure 3 
open reduction and internal fixation with plate.

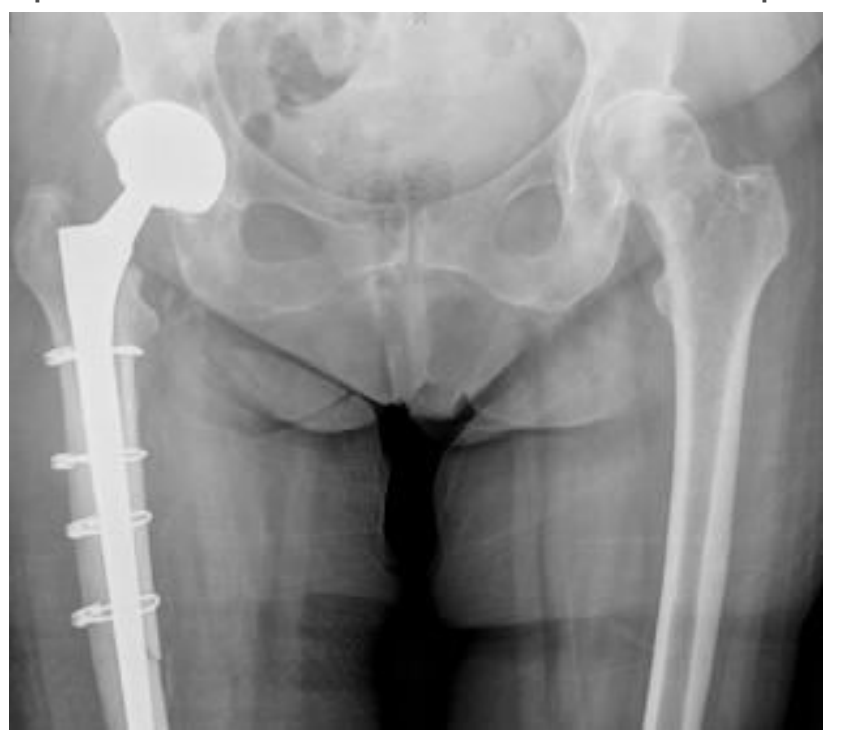

Figure 4

Revision arthroplasty. 